\title{
Modification of 5-Hydroxytryptophan-Evoked 5-Hydroxytryptamine Formation of Guinea Pig Colonic Mucosa by Reactive Oxygen Species
}

\author{
Shu-ichi Kojima ${ }^{1, *}$, Masashi Ikeda ${ }^{2}$, Asako Shibukawa $^{1}$ and Yuichiro Kamikawa ${ }^{1}$ \\ ${ }^{1}$ Department of Pharmacology and ${ }^{2}$ Institute of Medical Science, Dokkyo University School of Medicine, \\ Mibu, Tochigi 321-0293, Japan
}

Received August 23, 2001 Accepted October 25, 2001

\begin{abstract}
We studied whether reactive oxygen species (ROS) generated by normal colonic mucosa affect 5-hydroxytryptophan (5-HTP)-evoked 5-HT formation (measured as the sum of 5-HT plus 5-hydroxyindole acetic acid (5-HIAA) accumulation) of guinea pig's isolated colonic mucosa. Catalase (3000$6000 \mathrm{U} / \mathrm{ml}$ ), a hydrogen peroxide $\left(\mathrm{H}_{2} \mathrm{O}_{2}\right)$ scavenger or diphenylene iodonium (DPI, $10-100 \mu \mathrm{M}$ ), an NADPH oxidase inhibitor, concentration-dependently caused an increase of the sum of 5-HT plus 5-HIAA accumulation in the presence of 5-HTP $(10 \mu \mathrm{M})$, but these drugs did not significantly affect the 5-HT-metabolite in the colonic mucosa measured as the ratio of 5-HIAA/5-HT. Exogenously applied $\mathrm{H}_{2} \mathrm{O}_{2}(10-$ $100 \mu \mathrm{M})$ concentration-dependently inhibited the sum of 5-HT plus 5-HIAA accumulation. In contrast, neither superoxide dismutase (SOD, $100-300 \mathrm{U} / \mathrm{ml}$ ), superoxide anion scavenger, nor dimetyl sulfoxide (1-5\%, DMSO), a hydroxyl radical scavenger affected the sum of 5-HT plus 5-HIAA accumulation. Moreover, mucosa ROS generation was estimated using the chemiluminescence technique. SOD (100$300 \mathrm{U} / \mathrm{ml})$, catalase $(3000-6000 \mathrm{U} / \mathrm{ml})$ or DPI $(10-100 \mu \mathrm{M})$, concentration-dependently reduced luminolenhanced chemiluminescence signal from the colonic mucosa, while allopurinol $(10-100 \mu \mathrm{M})$, a xanthine oxidase inhibitor, did not affect the chemiluminescence signal. These results suggest that ROS is formed through an NADPH oxidase system in the guinea pig colonic mucosa, where it exerts a modulatory effect on mucosal 5-HT formation upon addition of 5-HTP. Thus, ROS formation from normal colonic mucosa could be considered to contribute to the control of 5-HT production in mucosa enterochromaffin cells.
\end{abstract}

Keywords: 5-Hydroxytryptamine, Colon (guinea pig), Enterochromaffin cell, 5-Hydroxytryptophan, Reactive oxygen species

5-Hydroxytryptamine (5-HT) has long been recognized as an important messenger substance involved in a wide variety of intestinal functions exerted through its interaction with multiple receptor subtypes (1), and in functional bowel disorders (2). Most of the intestinal 5-HT is produced and stored in mucosal enterochromaffin cells (EC cells) $(3,4)$ from which this amine is released into the intestinal lumen and the portal circulation $(5,6)$. The regulatory mechanism of 5-HT release from the EC cells has been studied more extensively, using a variety of experimental models in different animals $(7-9)$, but the regulation of 5 -HT production in the EC cells is not fully understood.

Reactive oxygen species (ROS), such as superoxide anion $\left(\mathrm{O}_{2}^{-}\right)$and hydrogen peroxide $\left(\mathrm{H}_{2} \mathrm{O}_{2}\right)$, are thought to be involved in the pathogenesis of experimental colitis in animal models and inflammatory bowel disease of humans
$(10-12)$, and in the defense against invading foreign organisms (13). The free radicals are released from various types of cells residing in intestinal mucosa. In this respect, neutrophils, as well as epithelial cells are known to be potent sources of these ROS (14). Normal colonic mucosa is also shown to be capable of producing $\operatorname{ROS}(15,16)$, but whether ROS produced by the normal colonic mucosa affect 5-HT production in the EC cells, is currently unclear. More recently, we have shown that the colonic mucosa of guinea pig is capable of converting the 5-HT precursor 5-hydroxytryptophan (5-HTP) to 5-HT (17). Therefore, the aim of the present study was to provide evidence for understanding the action of ROS on 5-HT production in colonic mucosa EC cells by elucidating the effects of ROS scavengers on 5-HTP-evoked 5-HT formation of guinea pig colonic mucosa.

*Corresponding author. FAX: +81-282-86-2915

E-mail: s-kojima@dokkyomed.ac.jp 


\section{MATERIALS AND METHODS}

\section{Tissue preparation}

Male Dunkin-Hartley guinea pigs of $250-300 \mathrm{~g}$ body weight were purchased from Shizuoka Laboratory Animal Center, Inc. (Shizuoka). Guinea pigs were anesthetized with enflurane and bled via the femoral artery. A segment of the proximal colon, $2-9.5-\mathrm{cm}$ away from the caecum was removed and the longitudinal/circular muscle layers were carefully stripped off as previously described (18). The underlying mucosa sheet and muscle layers were suspended in 15-ml tissue baths filled with modified Krebs solution $\left(120 \mathrm{mM} \mathrm{NaCl}, 4.7 \mathrm{mM} \mathrm{KCl}, 2.5 \mathrm{mM} \mathrm{CaCl}_{2}\right.$, $1.2 \mathrm{mM} \mathrm{MgCl}_{2}, 1.2 \mathrm{mM} \mathrm{KH}_{2} \mathrm{PO}_{4}, 25 \mathrm{mM} \mathrm{NaHCO}_{3}, 11 \mathrm{mM}$ glucose and $0.03 \mathrm{mM} \mathrm{Na} \mathrm{NDTA}_{2}$ ) at $37^{\circ} \mathrm{C}$ and bubbled with $95 \% \mathrm{O}_{2} / 5 \% \mathrm{CO}_{2}$. The tissues were then washed in modified Krebs solution for $60 \mathrm{~min}$ before the start of experiments. During the washing period, the bathing medium was replaced every $10 \mathrm{~min}$. The colonic mucosa was subsequently divided longitudinally in $7-9$ pieces for biochemical experiments.

\section{Measurement of 5-HT and 5-hydroxyindole acetic acid (5-HIAA)}

The mucosa pieces (approximately $20-50 \mathrm{mg}$ each) and muscle strips (1-cm-long) were suspended in 1.9-ml tissue baths containing preoxygenated Hank's balanced salt solution (HBSS, pH 7.4) kept at $37^{\circ} \mathrm{C}$. The HBSS mostly contained tetrodotoxin $(1 \mu \mathrm{M})$, a nerve conduction blocking agent and pyridoxal $(1 \mu \mathrm{M})$, a decarboxylase cofactor. After $5 \mathrm{~min}$ of preincubation, 5-HTP $(10-100 \mu \mathrm{M})$ was added into the medium. When the effects of scavengers /inhibitor or $\mathrm{H}_{2} \mathrm{O}_{2}$ were evaluated, the test drugs were added to the incubation medium from the start of incubation. After 90-min incubation with 5-HTP, 5-HT and 5-HIAA in the medium was measured by high performance liquid chromatography (HPLC) with electrochemical detection (LC-4B; BAS, West Lafayette, IN, USA) as described previously (9). The separation of 5-HT and 5-HIAA was achieved by a reverse-phase column (110-mm-long, 4-mm inner diater, C-18, $3 \mu \mathrm{m}$; BAS), using a mobile phase consisting of $0.1 \mathrm{M}$ monochloroacetic acid, $1 \mathrm{mM} \mathrm{Na} \mathrm{N}_{2}$ EDTA, $60 \mathrm{mg} / 1$ sodium octylsulfate and $10-15 \%$ acetonitrile (pH 3.2) at a flow rate of $0.4 \mathrm{ml} / \mathrm{min}$. 5-HTP-evoked 5-HT accumulation was expressed as nanomole per gram wet weight of tissue.

\section{Chemiluminescence studies}

Generation of ROS was measured by luminol-enhanced chemiluminescence as described previously $(16,19)$. To characterize the spontaneous release of free radicals from isolated colonic mucosa, the mucosa pieces were suspended in $1.9 \mathrm{ml}$ test tubes filled with HBSS containing either scavenger/inhibitor or vehicle at $37^{\circ} \mathrm{C}$. After $10 \mathrm{~min}$ of preincubation, the test tubes were placed in the luminescence reader (BLR-301; Aloka, Tokyo), and luminol $(100 \mu \mathrm{M})$ was added into the medium prior to measurement of chemiluminescence for $3 \mathrm{~min}$ at $37^{\circ} \mathrm{C}$. Chemiluminescence is expressed as a percentage of the control after subtraction of background (mean of photon counts detected by a test tube containing luminol and HBSS before and after each measurement from tissue suspension).

Drugs

The following drugs were used: tetrodotoxin (Sankyo, Tokyo); pyridoxal hydrochloride (Wako Pure Chemical, Osaka); benserazide hydrochloride (Research Biochemicals Inc., Natick, MA, USA); allopurinol, catalase, dimethyl sulfoxide, diphenylene iodonium (DPI), $\mathrm{H}_{2} \mathrm{O}_{2}, 5$-hydroxytryptophan, luminol, pyrogallol, superoxide dismutase (SOD) (Sigma Chemical Co., St. Louis, MO, USA). All drugs were initially dissolved in saline with the exception of luminol $(20 \mathrm{mM})$, which was dissolved in $0.1 \mathrm{M} \mathrm{NaOH}$.

\section{Statistical analyses}

Results are expressed as means \pm S.E.M. Statistical analysis was performed by ANOVA, followed by post hoc evaluation (according to Bonferroni or Dunnett where appropriate). A $P$ value $<0.05$ was considered significant.

\section{RESULTS}

\section{5-HTP-evoked 5-HT accumulation}

In the presence of tetrodotoxin $(1 \mu \mathrm{M})$ and pyridoxal $(1 \mu \mathrm{M})$, increasing concentration of 5-HTP (90-min treatment) in the incubation medium resulted in a concentrationdependent increase in the 5-HT accumulation of isolated colonic mucosa (Fig. 1). Furthermore, the increase in the 5HT accumulation in response to the highest concentration of 5-HTP used $(100 \mu \mathrm{M})$ was inhibited by coincubation of tissues with the amino acid decarboxylase inhibitor benserazide $(30 \mu \mathrm{M})$ (Fig. 1). In contrast, 5-HTP $(100 \mu \mathrm{M})$ was a poor amplifier of the 5-HT accumulation of the longitudinal/circular muscle preparations in the presence of pyridoxal $(0.16 \pm 0.07 \mathrm{nmol} / \mathrm{g}$ tissue, $\mathrm{n}=5$, Fig. 1$)$.

\section{Effects of scavengers/inhibitor}

To evaluate the possible effects of ROS on 5-HTP $(10 \mu \mathrm{M})$-evoked 5-HT formation (measured as the sum of 5-HT plus 5-HIAA accumulation), ROS scavengers and inhibitor were tested. After $90 \mathrm{~min}$ of incubation with 5HTP $(10 \mu \mathrm{M})$, accumulation levels of 5-HT and the 5-HT metabolite 5-HIAA was $6.0 \pm 0.6 \mathrm{nmol} / \mathrm{g}$ tissue and $0.8 \pm 0.2 \mathrm{nmol} / \mathrm{g}$ tissue $(\mathrm{n}=9)$, respectively. As shown in Fig. $2 \mathrm{~A}$, catalase $(3000-6000 \mathrm{U} / \mathrm{ml}), \mathrm{H}_{2} \mathrm{O}_{2}$ scavenger or DPI $(10-100 \mu \mathrm{M})$, an NADPH oxidase inhibitor, concen- 


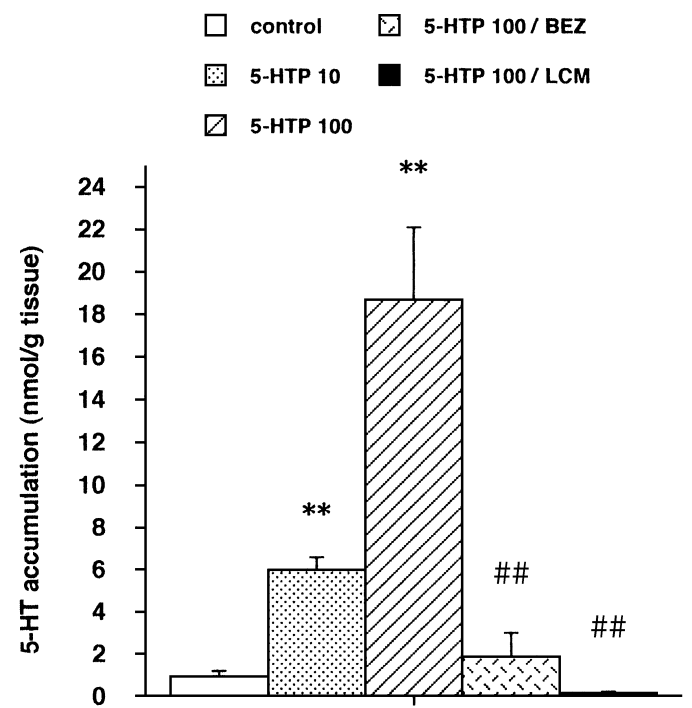

Fig. 1. 5-hydroxytryptophan (5-HTP, $10-100 \mu \mathrm{M}$ )-evoked 5-HT accumulation $(90 \mathrm{~min})$ of guinea pig colonic mucosa in the presence of tetrodotoxin $(1 \mu \mathrm{M})$ and pyridoxal $(1 \mu \mathrm{M})$, a decarboxylase cofactor. Some experiments were performed with the addition of the decarboxylase inhibitor benserazide (BEZ, $30 \mu \mathrm{M}$ ) or using longitudinal /circular muscle preparations (LCM) in the absence of tetrodotoxin. The 5-HTP-evoked 5-HT accumulation is expressed as nanomole per gram wet weight of tissue. Each column represents the mean and vertical lines show the S.E.M. of $5-9$ experiments. ${ }^{*} * P<0.01$ vs the control, ${ }^{\#} P<0.01$ vs the 5 -HTP $(100 \mu \mathrm{M})$ alone.

tration-dependently caused an increase of the sum of 5-HT and 5-HIAA accumulation in the presence of 5-HTP $(10 \mu \mathrm{M})$. In contrast, neither SOD $(100-300 \mathrm{U} / \mathrm{ml})$, a superoxide anion scavenger nor dimetyl sulfoxide $(1-5 \%$ DMSO), a hydroxyl radical scavenger affected the sum of 5-HT plus 5-HIAA accumulation.

In the presence of $\mathrm{H}_{2} \mathrm{O}_{2}(10-100 \mu \mathrm{M}, \mathrm{n}=4)$, the sum of 5 -HT plus 5-HIAA accumulation was significantly reduced by $82.2 \pm 3.8 \%$ and $56.6 \pm 6.0 \%$, respectively $(P<0.05)$. In contrast, pyrogallol $(100 \mu \mathrm{M})$, a superoxide anion donor, did not significantly affect the 5-HT/5-HIAA accumulation ( $\mathrm{n}=3$, data not shown).

Moreover, ROS scavengers was tested against 5-HTmetabolism (measured as the ratio between 5-HIAA/5-HT). Neither SOD $(300 \mathrm{U} / \mathrm{ml})$, catalase $(6000 \mathrm{U} / \mathrm{ml})$ nor $5 \%$ DMSO affected the 5-HIAA/5-HT ratio (Fig. 2B). DPI $(100 \mu \mathrm{M})$ showed a tendency to reduce the ratio of 5HIAA/5-HT, but these changes did not reach statistical significance (Fig. 2B).

\section{Chemiluminescence studies}

The mucosa pieces suspended in buffer produced a measurable chemiluminescence signal. SOD $(100-300 \mathrm{U}$ $/ \mathrm{ml})$, catalase $(3000-6000 \mathrm{U} / \mathrm{ml})$ or DPI $(10-100 \mu \mathrm{M})$ concentration-dependently reduced the luminol-enhanced chemiluminescence signal from the colonic mucosa (Fig. 3).
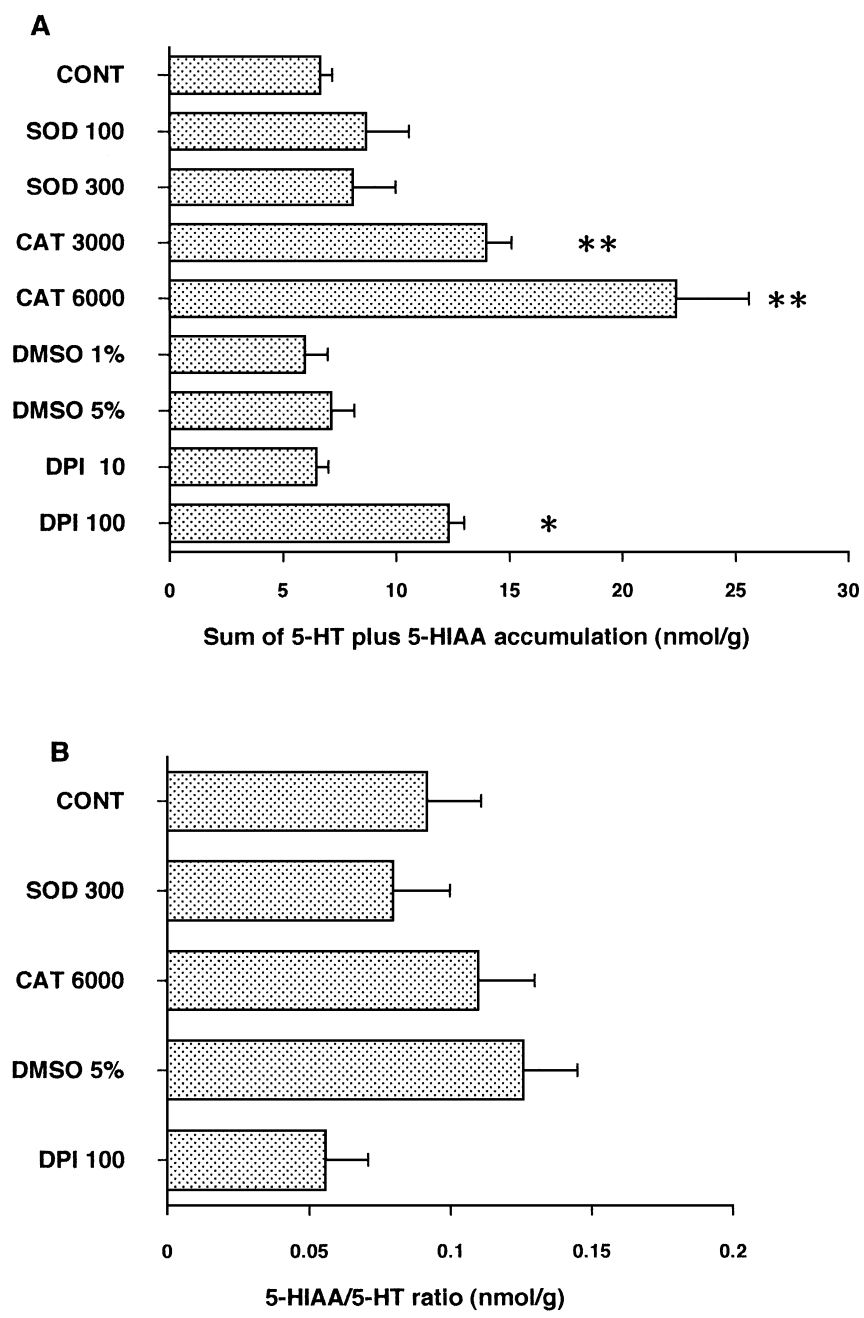

Fig. 2. Effects of ROS scavengers/inhibitor on 5-HTP $(10 \mu \mathrm{M})$ evoked 5-HT formation in the guinea pig colonic mucosa measured as the sum of 5-HT plus 5-HIAA accumulation (A) or on 5-HTmetabolite in the colonic mucosa measured as the 5-HIAA/5-HT ratio (B). Superoxide dismutase (SOD, $100-300 \mathrm{U} / \mathrm{ml}$ ), catalase (CAT, $3000-6000 \mathrm{U} / \mathrm{ml}$ ), dimetyl sulfoxide (DMSO, $1-5 \%$ ) and diphenylene iodonium (DPI, $10-100 \mu \mathrm{M}$ ), were added to the incubation medium from the start of incubation. Tetrodotoxin $(1 \mu \mathrm{M})$ and pyridoxal $(1 \mu \mathrm{M})$ were present in all experiments. Each column represents the mean and vertical lines show S.E.M. of 5-9 experiments. ${ }^{*} P<0.05$, $* * P<0.01$ vs the control (CONT).

In contrast, allopurinol $(10-100 \mu \mathrm{M})$, a xanthine oxidase inhibitor, did not affect the chemiluminescence signal (Fig. 3). Longitudinal/circular muscle preparations did not produce a chemiluminescence signal $(n=4$, data not shown $)$.

\section{DISCUSSION}

The present study deals with the question of whether ROS generated by the colonic mucosa affect mucosal 5-HT formation upon addition of 5-HTP, the endogenous 5-HT precursor. Under the present in vitro conditions (in the 


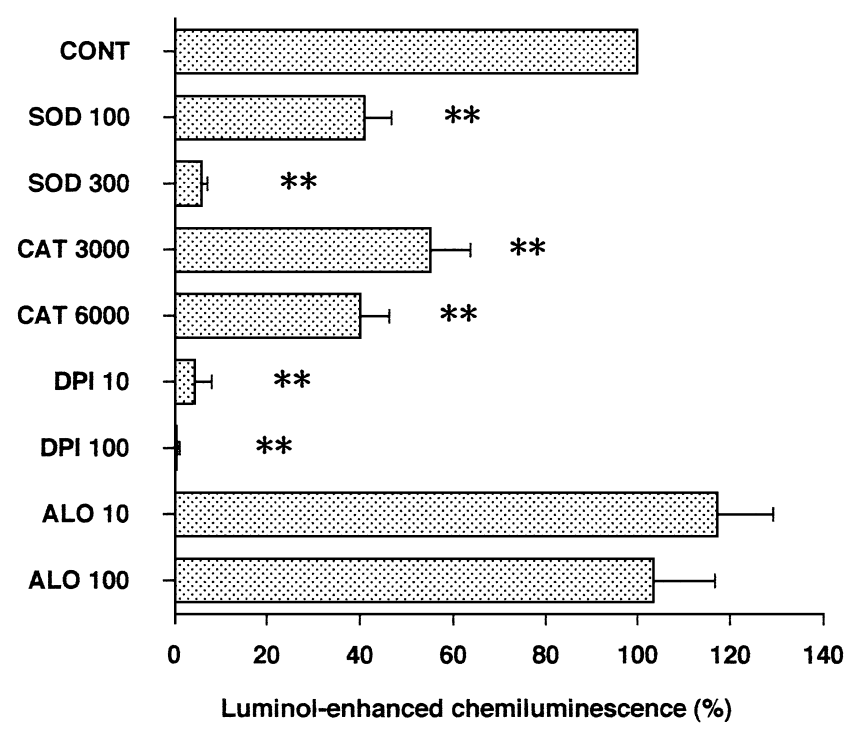

Fig. 3. Effects of ROS scavengers/inhibitor on luminol (100 $\mu \mathrm{M})$ enhanced chemiluminescence from the colonic mucosa. SOD $(100-300 \mathrm{U} / \mathrm{ml})$, catalase (CAT, $3000-6000 \mathrm{U} / \mathrm{ml})$, diphenylene iodonium (DPI, $10-100 \mu \mathrm{M}$ ) and allopurinol (ALO, $10-100 \mu \mathrm{M}$ ), were added to the incubation medium from the start of incubation. The luminol-enhanced chemiluminescence in the presence of test drugs is expressed as a percentage of the control (CONT). Each column represents the mean and the vertical line shows the S.E.M. of $5-7$ experiments. ${ }^{* *} P<0.01$ vs the control.

presence of tetrodotoxin), 5-HTP concentration-dependently increased the 5-HT accumulation in the isolated guinea pig colonic mucosa, which closely correlates with changes in the 5-HTP-evoked luminal 5-HT release from the colon (17), whereas 5-HTP was a poor amplifier of the 5-HT accumulation of the longitudinal/circular muscle preparations with adherent myenteric plexus. Thus, these results suggest that 5-HTP-evoked 5-HT accumulation in this preparation reflects almost exclusively $5-\mathrm{HT}$ production from non-neuronal 5-HT-producing cells in the colonic mucosa. This conclusion is in keeping with the notion that no 5-HT-immunoreactive neurons in the guinea pig colonic mucosa is found (20). The EC cells have commonly been addressed, as a candidate of the 5-HT-producing cells in the colonic mucosa. In addition, we recently reported (17) that using the luminally perfused isolated guinea pig colon, 5-HTP facilitates the luminal 5-HT release from EC cells. Thus, it is possible that the 5-HTP-evoked 5-HT formation occurs in EC cells, although the amount of the 5-HTPevoked 5-HT formation in part contains that due to "epithelial cells" contamination (7). If 5-HT is produced also by the epithelial cells, the function of that 5-HT compared to that produced by the EC cells is largely unknown.

To determine the influence of ROS on the enhanced 5HT formation by 5-HTP, we examined the effects of catalase, a $\mathrm{H}_{2} \mathrm{O}_{2}$ scavenger; $\mathrm{SOD}, \mathrm{O}_{2}^{-}$scavenger; and DMSO, a hydroxyl radical scavenger. In this study, we found that 5-HT formation (measured as the sum of 5-HT plus 5HIAA accumulation) of the colonic mucosa evoked by 5HTP was enhanced in the presence of catalase, suggesting a role for hydrogen peroxide as a modulator of 5-HT biosynthesis. Furthermore, the possibility that $\mathrm{H}_{2} \mathrm{O}_{2}$ may affect 5HT degradation processes is unlikely, because catalase had no effect on 5-HT-metabolism (measured as the ratio between 5-HIAA/5-HT). In contrast, SOD and DMSO failed to modify the 5-HT formation in response to 5-HTP. The potency of SOD or DMSO used in the present study has been well documented $(10,12,21)$. Thus, it seems likely that neither $\mathrm{O}_{2}^{-}$nor a $\mathrm{H}_{2} \mathrm{O}_{2}$ hydrogen peroxide metabolite, hydroxyl radical, participated in the modification of the enhanced 5-HT formation.

Furthermore, we have examined whether ROS are generated from the colonic mucosa using the chemiluminescence technique. In the present study, we showed that the colonic mucosa fragments could produce a measurable luminol-enhanced chemiluminescence signal. The marked decrease in chemiluminescence signal caused by SOD and the NADPH oxidase inhibitor DPI suggests that superoxide anion predominantly via an NADPH oxidase system is generated from the colonic mucosa. The partial decrease in the chemiluminescence signal caused by catalase also suggests that $\mathrm{H}_{2} \mathrm{O}_{2}$ is one of the ROS detected in the colonic mucosa. These findings are consistent with previous data showing that the normal rat colonic mucosa (15) or the normal guinea pig gastric mucosa (22) spontaneously generates superoxide anion. Nevertheless, SOD failed to modify the enhanced 5-HT formation by 5-HTP. Such data implies that $\mathrm{O}_{2}{ }^{-}$may be only useful as a precursor of the more stable ROS, $\mathrm{H}_{2} \mathrm{O}_{2}$. In the present study, we also observed that increasing concentrations $(10-100 \mu \mathrm{M})$ of $\mathrm{H}_{2} \mathrm{O}_{2}$ in the incubation medium resulted in a decrease in the enhanced 5-HT production. Hence, it is considered that the enhancement of the 5-HT formation to 5-HTP by catalase is due to the degradation of the enhanced extracellular $\mathrm{H}_{2} \mathrm{O}_{2}$, resulting from the dismutation of superoxide anion. There are various possible sources of ROS production in the colonic mucosa; they include xanthine oxidase within epithelial cells and NADPH oxidase in phagocytic leukocytes (23). In our experiments, xanthine oxidase does not contribute substantially to the ROS production as evidenced by a lack of effect of allopurinol, a xanthine oxidase inhibitor, on the chemiluminescence signal. Moreover, it is well known that neutrophils are abundantly present in the inflamed colonic mucosa, but not in the normal colonic mucosa $(10,16)$. In accordance with the effect of catalase, DPI also enhanced the 5-HT formation evoked by 5-HTP. This probably represents the role of NADPH oxidase system in regulating 5HT levels of the colonic mucosa. Recently Suh et al. (24) showed that the superoxide-generating NADPH oxidase 
Mox 1 messenger RNA is expressed most in normal human colon, but not in peripheral blood leukocytes. Thus, a superoxide-generating NADPH oxidase Mox1-like system might contribute to the superoxide generation from the colonic mucosa, as a possible source.

In conclusion, the results of the present study provide evidence that ROS is formed through an NADPH oxidase system in the guinea-pig colonic mucosa, where it exerts a modulatory effect on mucosal 5-HT formation upon addition of 5-HTP. Thus, ROS formation from normal colonic mucosa could be considered to contribute to the control of 5 -HT production in mucosa enterochromaffin cells.

\section{Acknowledgment}

This work was supported in part by a grant from the Japan Health Science Foundation, Tokyo.

\section{REFERENCES}

1 Gershon MD: Roles played by 5-hydroxytryptamine in the physiology of the bowel. Aliment Pharmacol Ther 13, 15-30 (1999)

2 Sanger GJ: 5-Hydroxytryptamine and functional bowel disorders. Neurogastroenterol Motil 8, 319 - 331 (1996)

3 Erspamer $\mathrm{V}$ and Asero B: Identification of enteramine, the specific hormone of the enterochromaffin cell system, as 5hydroxytryptamine. Nature 169, 800 - 801 (1952)

4 Larsson LI and Jorgensen LM: Ultrastractural and cytochemical studies on the cytodifferentiation of duodenal endocrine cells. Cell Tissue Res 194, 79 - 102 (1978)

5 Bulbring E and Lin RCY: The effect of intraluminal application of 5-hydroxytryptamine and 5-hydroxytryptophan on peristalsis; the local production 5-HT and its release in relation to intraluminal pressure and propulsive activity. J Physiol (Lond) 140, $381-407$ (1958)

6 Schwörer H, Racké K and Kilbinger H: Spontaneous release of endogenous 5-hydroxytryptamine and 5-hydroxyindoleacetic acid from the isolated vascularly perfused ileum of the guineapig. Neuroscience 21, 297 - 303 (1987)

7 Racké K, Reimann A, Schwörer H and Kilbinger H: Regulation of 5-HT release from enterochromaffin cells. Behav Brain Res 73, 83 - 87 (1996)

8 Fujimiya M, Okumiya $\mathrm{K}$ and Kuwahara A: Immunoelectron microscopic study of the luminal release of serotonin from enterochromaffin cells induced by high intraluminal pressure. Histochem Cell Biol 108, 105 - 113 (1997)

9 Kojima S and Ikeda M: Facilitation by endogenous acetylcholine and nitric oxide of luminal serotonin release from the guinea-pig colon. Eur J Pharmacol 355, 51 - 55 (1998)
10 Simmonds NJ, Allen RE, Stevens TRJ, Van Someren RNM, Blake DR and Rampton DS: Chemiluminescence assay of mucosal reactive oxygen metabolites in inflammatory bowel disease. Gastroenterology 103, 186 - 196 (1992)

11 Mckenzie SJ, Baker MS, Buffinton GD and Doe WF: Evidence of oxidant-induced injury to epithelial cells during inflammatory bowel disease. J Clin Invest 98, 136 - 141 (1996)

12 Qiu B, Pothoulakis C, Castagliuolo I, Nikulasson S and Lamont JT: Participation of reactive oxygen metabolites in Clostridium difficile toxin A-induced enteritis in rat. Am J Physiol 276, G485 - G490 (1999)

13 Mahida YR, Wu KC and Jewell DP: Respiratory burst activity of intestinal macrophages in normal and inflammatory bowel disease. Gut 30, 1362 - 1370 (1989)

14 Nilsson UA, Schoenberg NH, Aneman A, Poch B, Magadum S, Beger $\mathrm{HG}$ and Lundgren $\mathrm{O}$ : Free radicals and pathogenesis during ischemia and reperfusion of the cat small intestine. Gastroenterology 106, 629-636 (1994)

15 Craven PA, Pfanstiel J and Derubertis FR: Role of reactive oxygen in bile salt stimulation of colonic epithelial proliferation. J Clin Invest 77, 850 - 859 (1986)

16 Keshavarzian A, Sedghi S, Kanofsky J, List T, Robinson C, Ibrahim $\mathrm{C}$ and Winship D: Excessive production of reactive oxygen metabolites by inflamed colon: analysis by chemiluminescence probe. Gastroenterology 103, 177 - 185 (1992)

17 Kojima S, Ikeda M and Kamikawa Y: Investigation into the 5hydroxytryptophan-evoked luminal 5-hydroxytryptamine release from the guinea-pig colon. Jpn J Pharmacol 84, 174 - 178 (2000)

18 Kojima S and Shimo Y: An enhancing effect of 5-hydroxytryptamine on electrically evoked atropine-resistant contraction of guinea-pig proximal colon. Br J Pharmacol 114, $73-76$ (1995)

19 Dahlgren C and Karlsson A: Respiratory burst in human neutrophils. J Immunol Methods 232, 3 - 14 (1999)

20 Wardell CF, Bornstein JC and Furness JB: Projection of 5hydroxytryptamine-immunoreactive neurons in guinea-pig distal colon. Cell Tissue Res 278, 379 - 387 (1994)

21 Kennedy S, Work L, Ferris P, Miller A, Mcmanus B, Wadsworth $\mathrm{RM}$ and Wainwright CL: Role of nitric oxide and free radicals in the contractile response to non-preactivated leukocytes. Eur J Pharmacol 345, 269 - 277 (1998)

22 Teshima S, Rokutan K, Nikawa T and Kishi K: Guinea-pig gastric mucosal cells produce abundant superoxide anion through an NADPH oxidase-like system. Gastroenterology $\mathbf{1 1 5}$, 1186 - 1196 (1998)

23 Predue MH and Mckay DM: Integrative immunophysiology in the intestinal mucosa. Am J Physiol 267, G151 - G165 (1994)

24 Suh YA, Arnold RS, Lassegue B, Shi J, Xu X, Sorescu D, Chung $\mathrm{AB}$, Griendling KK and Lambeth JD: Cell transformation by the superoxide-generating oxidase Mox1. Nature 401, 79-82 (1999) 